\title{
Microangiopathic haemolytic anaemia: The phenomenon of red cell adherence
}

\author{
F. E. PRESTON AND R. CHRISTENHUSZ 1
}

From the University Department of Haematology, Royal Infirmary, Sheffield, S6 3DA, England, and the Institute of Medical Physics, Wilhelms University, Munster, Westphalia, West Germany

SYNOPSIS Examination by scanning electron microscopy of erythrocytes from three cases of $Z$ microangiopathic haemolytic anaemia demonstrated the presence of adherent cells. Although in some cases this appeared to be due to a thin strand, presumably fibrin, between the erythro- $\bar{\partial}$ cytes, in most instances there was intimate contact and this was invariably associated with $\stackrel{\mathbb{}}{\stackrel{D}{ }}$ damage to the red cell surface membrane.

It is considered that the adherence is predominantly due to altered physico-chemical pro-. perties of the surface membrane of the red blood cell and that further morphological abnormalities may result from the intravascular stresses to which the paired cells are subjected.

Microangiopathic haemolytic anaemia is a haemolytic disorder secondary to disease of small blood vessels and is characterized by the presence of fragmented and distorted erythrocytes in the peripheral blood (Brain, Dacie, and Hourihane, 1962). Of particular interest is its association with disseminated intravascular coagulation (Bull and Brain, 1968), a pathological process which may be triggered off by a number of aetiological mechanisms such as release of tissue thromboplastin, anoxia, anoxaemia, and endothelial damage (McKay, 1968). The cause of the distortion and fragmentation of red blood cells which are such constant features of microangiopathic haemolytic anaemia is considered to be mechanical trauma occurring within small blood vessels (Brain et al, 1962; Bull and Brain, 1968; Bull, Rubenberg, Dacie, and Brain, 1968).

In the present study scanning electron microscopy was used to examine blood from three cases of microangiopathic haemolytic anaemia. Adherent erythrocytes were readily demonstrated in each case and evidence is presented to show how this phenomenon may result in further destruction of the red blood cell.

'Present address: Geologico-palaentological Institute, The University, Marburg Lahn, West Germany.

Received for publication 8 March 1970.

\section{Materials and Methods}

Specimens of venous blood were taken directly? into $0.5 \%$ phosphate-buffered glutaraldehyde $(p \mathrm{H} \mathrm{7.4)}$ at room temperature. After 15 to 30 \% minutes' fixation they were centrifuged at 5003 revs/min for five minutes, and then washed six times in double-distilled water. One drop was placed on a coverslip and allowed to dry in $a_{\circ}$ dust-free atmosphere. After coating the dried specimens with a thin film of gold they were examined with the Cambridge Stereoscan electron microscope.

\section{SUBJECTS STUDIED}

Ten specimens from eight normal subjects and five specimens from three patients with micro- 0 angiopathic haemolytic anaemia were studied: $\mathbb{\oplus}$ brief details of the latter are as follows.

\section{Case 1}

Acute onset of unknown aetiology associated with acute renal failure and peripheral vein throm- $\mathbb{D}$ bosis.

Case 2

Insidious onset in a case of longstanding rheumatoid arthritis. 


\section{Case 3}

Chronic renal failure and hypertension.

In all three patients the criteria for diagnosis of microangiopathic haemolytic anaemia were: (a) the presence of burr cells and schistocytes in the peripheral blood; (b) a reticulocyte count of $5 \%$ or more; and (c) a haemoglobin less than 9.0 $\mathrm{g} / 100 \mathrm{ml}$.

\section{Results}

\section{NORMAL CONTROLS}

The erythrocytes were regular, biconcave discs 7 to $8 \mu$ in diameter. Anisocytosis was minimal and poikilocytosis absent. The surface of the mature erythrocyte was smooth and featureless. A small proportion of the red blood cells possessed a solitary papillomatous-like projection directed into the lumen of the cell. The erythrocytes were evenly distributed on the preparation and showed no tendency to clump (Fig. 1).

MICROANGIOPATHIC HAEMOLYTIC ANAEMIA As expected, many bizarre forms were seen. In contrast to the even distribution of erythrocytes from normal subjects, red blood cells from cases of microangiopathic haemolytic anaemia showed a tendency to irregular clumping (Fig. 2).

In addition to the gross morphological abnormalities many of the red blood cells had an abnormal surface membrane. Particularly striking in case 2 were large numbers of smaller than normal spheroidal cells in which the membrane was irregularly heaped up, giving an appearance $\frac{\rho}{\overline{5}}$ not unlike a ball of wool (Fig. 3). Although these cells resembled lymphocytes they were, in fact, $\overrightarrow{0}$ smaller and, moreover, they constituted about $6 \%$ of the total erythrocyte population which $\underset{\vec{F}}{\vec{*}}$ corresponded to the proportion of spherocytes inthis patient's peripheral blood. Since the diameter $\frac{-}{0}$ and general morphology were also comparable $\overline{\bar{n}}$ it is considered, therefore, that they are the $\widehat{\otimes}$ spherocytes of microangiopathic anaemia.

In addition to a number of tears and membrane ${ }^{\infty}$ defects, observed in specimens from all three $\vec{\circ}$ cases, the surface of some erythrocytes was $\overrightarrow{-}$ characterized by coarse corrugations whilst in $\bar{\omega}$ others this was thrown into very many small, irregular, bleb-like eruptions (Fig. 4).

Adherent cells were noted in all specimens from patients with microangiopathic haemolytic anae- $\infty$ mia. They were not seen in any of the preparations from normal control subjects. Various modes of adherence were observed and tearing of the surface membrane at points of contact was $Z$ readily demonstrated. In most instances the affected cells were intimately opposed to each other (Fig. 5), but some cells were joined by음 spinous processes which occasionally resulted in a torn membrane (Fig. 6). This type was particu- $\overrightarrow{0}$ larly prominent in case 1 . Furthermore, a few cells were joined by thin intercellular strandso which did not appear to be derived from either surface membrane. This was observed chiefly in case 3.

Further examination of the five specimens $\frac{\circ}{\circ}$ demonstrated that projections from the surface of erythrocytes may be formed by the separation of adherent cells.

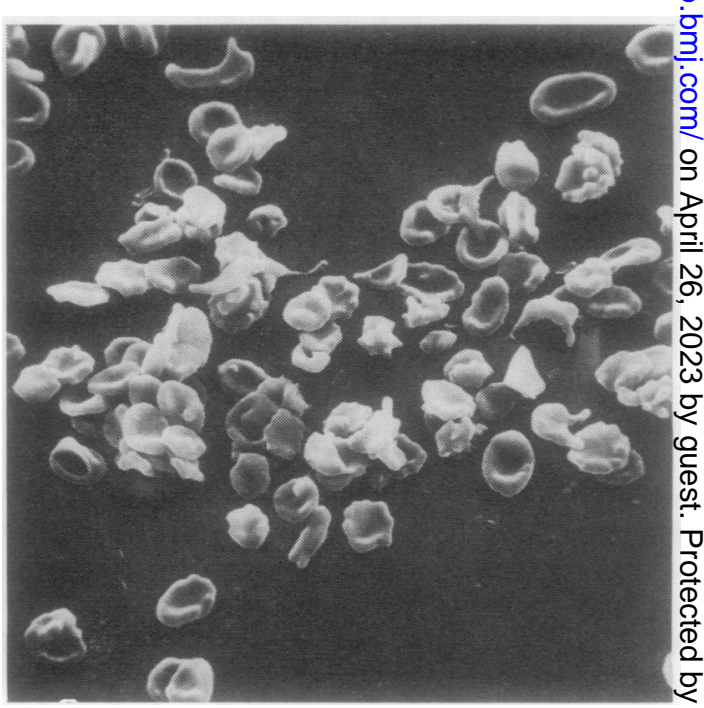

Fig. 2 Scanning electron microscopy, showing clumping of erythrocytes in microangiopathic haemolytic anaemia $(\times 1,200)$.
Fig. 1 Scanning electron microscopy, showing even distribution of erythrocytes from a normal control

subject $(\times 1,300)$.

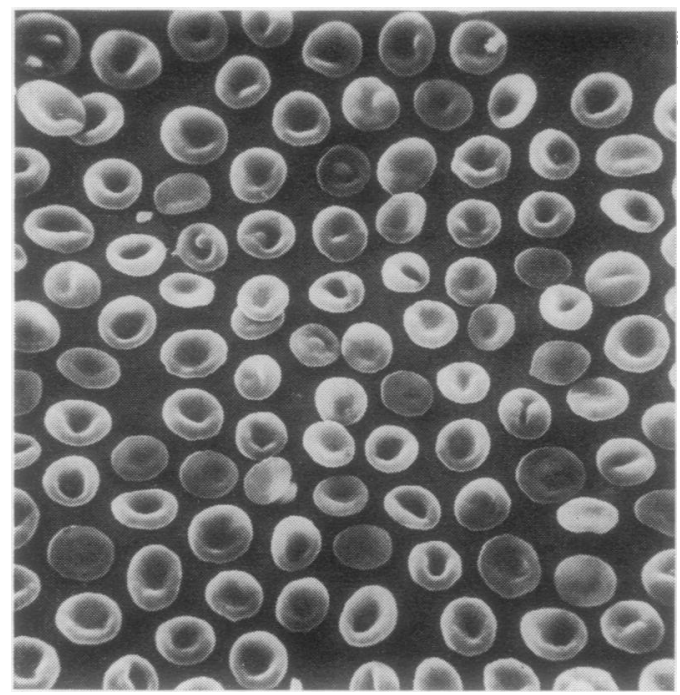




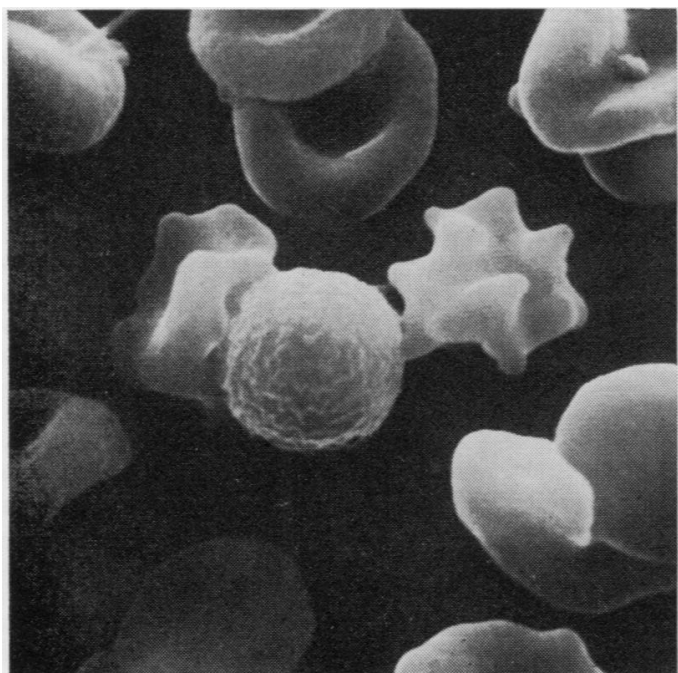

Fig. 3 Scanning electron microscopy from case 2, showing typical 'wool-ball' appearance of spherocytes $(\times 5,400)$.

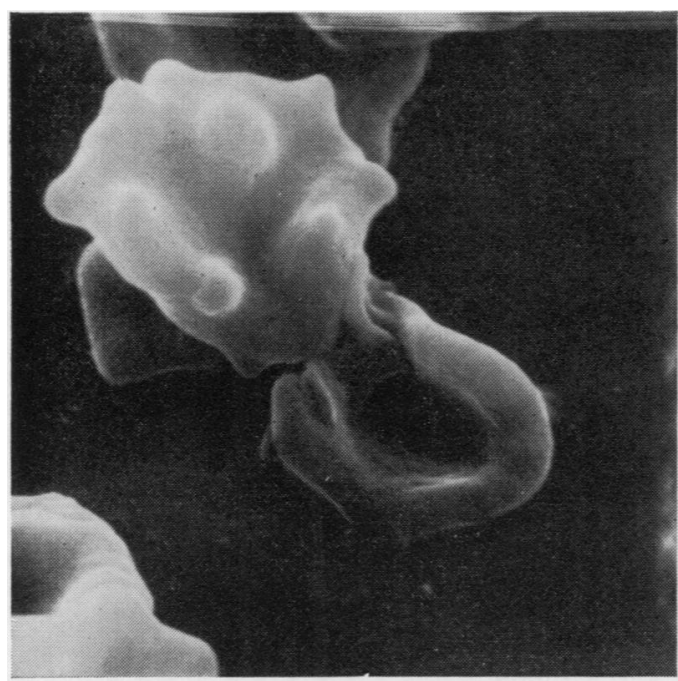

Fig. 5 Scanning electron microscopy from case 2, showing adherent erythrocytes with cell membrane damage $(\times 7,700)$.

\section{Discussion}

Many different terms have been applied to the abnormal distorted erythrocytes found in certain haemolytic anaemias. Thus 'burr cells', 'spicule cells', 'helmet cells', and 'triangular cells' are frequently described. A feature which is common to all of these is the presence of spiny projections extending from some point on the periphery. Ehrlich (1891), who first described these abnormal erythrocytes, considered them to be the result of circulating chemical substances secondary to anaemia, but within recent years attention has

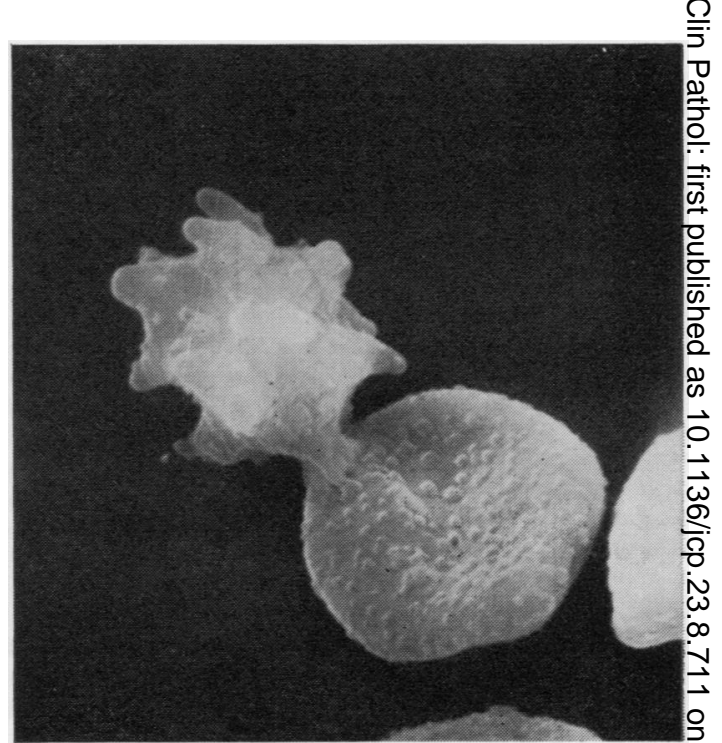

Fig. 4 Scanning electron microscopy from case 1, showing adherent erythrocytes and abnormal red cell surface membrane $(\times 6,700)$.

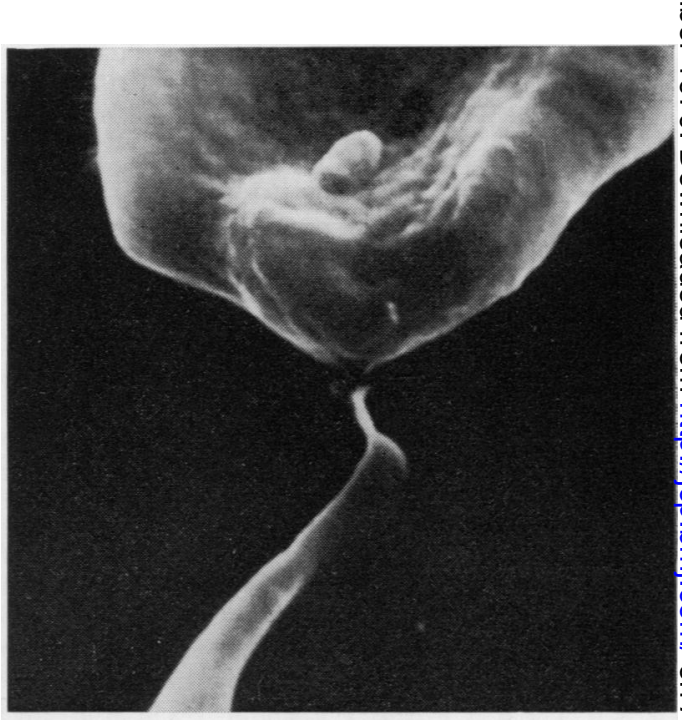

Fig. 6 Scanning electron microscopy from case 3, showing tearing of red cell membrane by spinous process of an adherent cell $(\times 15,200)$.

been focused on mechanical factors. Following a study on patients with microangiopathico haemolytic anaemia, Brain et al (1962) considered $\frac{c}{\Phi}$ that the common link between the haemolytic? anaemia and the underlying disease was the $\square$ presence of pathological changes in small blood $\underset{+}{+}$ vessels. They suggested that an important factor $\stackrel{\mathbb{D}}{\square}$ in the pathogenesis of the haemolysis is direct $\stackrel{\mathbb{Q}}{\Omega}$ contact between red cells and the diseased vesselo wall. More recently, Bull and Brain (1968) have demonstrated that red cells can fragment when a rapidly moving erythrocyte encounters a thin fibrin strand. 
It appears to be fairly well accepted that contact between erythrocytes and diseased blood vessels is of prime importance in the production of the abnormal erythrocytes of microangiopathic haemolytic anaemia. However, it has not been shown previously that red cell destruction may continue after the initial damage has taken place, although Venkatachalam, Jones, and Nelson (1968) observed frequent close juxtaposition of erythrocytes to endothelial cells in experimentally produced microangiopathic haemolytic anaemia in rats. These authors suggested that red cells may become adherent to endothelium and then fragment as a result of shearing stresses. In another report of experimentally produced microangiopathic haemolytic anaemia in rabbits, Rubenberg, Regoeczi, Bull, Dacie, and Brain (1968) observed that erythrocytes in wet preparations tended to adhere to each other and also to glass surfaces. They concluded that this effect was mediated through fibrin which had become attached to one of the red cell surfaces.

Salisbury and Clarke (1967) have demonstrated the potential of the scanning electron microscope in the study of erythrocytes. This instrument, which has greater depth of focus and higher resolution than the light microscope, has proved of value in demonstrating pathological changes, particularly of the red cell surface membrane.

In this study examination by scanning electron microscopy revealed erythrocytes connected in a variety of ways. In one subject only some cells were joined by a thin strand passing between them. Although contamination of the preparation cannot definitely be excluded, these strands were not observed in any of the control specimens; neither have they been seen in other conditions. It is believed that they are most probably fibrin. The majority of adherent cells, however, were in intimate contact and this was invariably associated with some alteration of the surface membrane. The third type of adherence was by means of spinous processes which appeared to be formed by the separation of cells which were only connected by a small area of surface membrane. On occasion this resulted in a torn surface membrane. In neither of the last two modes of adherence was there any evidence of interposed fibrin.

The negative electrical charge at the surface of normal erythrocytes ensures their functional and structural independence within the circulation. Alteration in the physico-chemical properties of the surface membrane of the red cell may result in the loss of the surface charge and the associated natural repulsion between cells. It is perhaps significant that both fibrinogen and globin, a histone, can increase the adhesiveness of erythrocytes (Ponder, 1966). The importance of fibrin in the pathogenesis of this condition has already been mentioned, but the presence of free haemo- globin, released from erythrocytes in the affected vessels, may also contribute to the observed $\stackrel{0}{ \pm}$ phenomenon.

The resolution of the scanning electron micro $\because$ scope is inadequate for the demonstration ofos? antibodies which may be present on the surfaceo membrane. The direct antihuman globulin tests, 흠 however, were repeatedly negative in the three $\frac{\bar{\rho}}{\text {. }}$ patients and red cell antibodies are not considered $\stackrel{\mathbb{Q}}{\Omega}$ a feature of this condition. A small proportion of cells were connected by fibrin strands, presumably acquired during passage through the affected. vessels. Apart from these, the most likely expla- $\overrightarrow{\vec{\omega}}$ nation of the phenomenon of adherence between $\omega$ erythrocytes is alteration of the physico-chemicalo properties of the red cell surface membrane with ain consequent increase in physical affinity between cells.

Although it is accepted that many of the abnor- $\overrightarrow{-}$ mal cells observed in microangiopathic haemolytico anaemia reflect mechanical trauma occasionedwithin the damaged vasculature, it is apparent $Z$ that further red cell damage may occur when adherent erythrocytes are subjected to the stresses of the vascular flow, and that, in some cases, these forces are sufficient to break the contact at its weakest point with the formation of the spicules which are a feature of this condition.

We wish to thank Dr E. K. Blackburn, University» Department of Haematology, Sheffield, England, $\stackrel{\mathbb{Q}}{\circ}$ for valuable assistance in the preparation of this $\Rightarrow$ manuscript. We also thank the Deutsche 3 Forschungsgemeinschaft for the use of the? Cambridge Stereoscan electron microscope.

References

Brain, M. C. Dacie, J. V and Hourihane, D. O'B. (1962) Microangiopathic haemolytic anaemia: the possible role of vascular lesions in pathogenesis. Brit. J. Haemat., 8, 358-374.

Bull, B. S., and Brain, M. C. (1968). Experimental models of microangiopathic haemolytic anaemia. Proc. roy. Soc. D Med., 61, 1134-1137.

Bull, B. S., Rubenberg, M. L., Dacie, J. V., and Brain, M. C. (1968). Microangiopathic haemolytic anaemia: mechanisms of red-cell fragmentation: in vitro studies. Brit. J. Haemat., 14, 643-652.

Ehrlich, P.(1891). Farbenanalytische Untersuchungen zur Histologie und Klinik des Blutes, vol. 1, p. 104. Hirschwald, Berlin. $\omega$

McKay, D. G. (1968). Pathology, diagnosis and therapy of dis- $\sigma$ seminated intravascular coagulation. Proc. roy. Soc. Med., 61, $1129-1134$.

Ponder, E. (1966). Effect of basic proteins on the adhesiveness of red cells. Nature (Lond.), 209, 307-308.

Rubenberg, M. L., Regoeczi, E., Bull, B. S., Dacie, J. V., and Brain, M. C. (1968). Microangiopathic haemolytic anae- $\square$ mia: the experimental production of haemolysis and red- $\bar{O}$

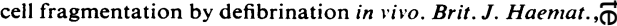
14, 627-642.

Salisbury, A. J., and Clarke, J. A. (1967). New method for $\vec{D}$ detecting changes in the surface appearance of human red blood cells. J. clin. Path., 20, 603-610.

Venkatachalam, M. A., Jones, D. B., and Nelson, D. A. (1968). Microangiopathic hemolytic anemia in rats with malignanto hypertension. Blood, 32, 278-291. 